\title{
I. TENEUR EN ACIDES AMINES ET VALEUR NUTRITIVE DES ALIMENTS
}

\author{
I N T R O D U C T I O N
}

\author{
G. FAUCONNEAU \\ Laboratoire des Métabolismes, \\ Centre national de Recherches zootechniques, Fouy-en-fosas (Seine-et-Oise).
}

La composition en acides aminés indispensables et semi-indispensables des protéines alimentaires détermine en grande partie leur valeur nutritive $(73 \%$ de variance contrôlée); aussi est-il intéressant de connaitre avec précision la composition en acides aminés des différentes protéines utilisées dans l'alimentation de l'homme et des animaux.

Les méthodes modernes de fractionnement des acides aminés par chromatographie sur colonne permettent de doser avec précision après hydrolyse acide, la plupart des acides aminés indispensables et semi-indispensables. L'utilisation d'appareils entièrement automatiques permet d'améliorer la précision (plus ou moins $2 \%$ ) et la finesse de séparation.

Seule la cystine et le tryptophane ne peuvent être dosés grâce à cette méthode : la cystine peut être déterminée avec précision après transformation en acide cystéique au sein de la protéine, hydrolyse et fractionnement sur colonne d'échangeur d'anions ou de cations (cf. de Belsunce, Pion, 1963, Ann. Biol. anim. Bioch. Biophys.). Les résultats obtenus grâce à cette méthode sont souvent différents de ceux obtenus par voie microbiologique (Cf. PION et al., ci-dessous).

La détermination du tryptophane fait l'objet d'études en cours : l'hydrolyse alcaline permet d'obtenir l'acide aminé libre qui est fractionné sur colonne d'Amberlite IR $120(90 \times 0,9 \mathrm{~cm})$. La séparation de composés interférents paraît difficile dans certains échantillons particulièrement pauvres en tryptophane et riches en certains glucides (mais), l'addition d'alcool benzylique, permettant la sortie plus rapide du tryptophane améliore un peu la séparation. Dans les protéines alimentaires où la teneur en tryptophane dépasse $1,1 \%$ (pour $16 \mathrm{~g}$. d'azote) la détermination paraît possible avec une précision satisfaisante. 